\title{
The Galactic path of stellar black holes and neutron stars
}

\author{
Irapuan Rodrigues ${ }^{1,2}$ and I. Félix Mirabel ${ }^{2,3}$ \\ ${ }^{1}$ Instituto de Física, Universidade Federal do Rio Grande do Sul, CP 15001, 91501-970, Porto \\ Alegre, Brazil email: irapuan@if.ufrgs.br \\ ${ }^{2}$ Service d'Astrophysique, CEA-Saclay, 91191 Gif-sur-Yvette, France email: fmirabel@cea.fr \\ ${ }^{3}$ Instituto de Astronomía y Física del Espacio/Conicet. Bs As, Argentina
}

\begin{abstract}
The kinematics of black hole and neutron star X-ray binaries in the Galaxy should help to know their birth place and constrain their evolution. We have used multiple tools of modern astronomy to determine the trajectories in the Galaxy and track the origins of black hole and neutron star X-ray binaries that are of topical interest in astrophysics. We find three distinct classes of black hole and neutron star X-ray binaries: (1) low mass X-ray binaries that move at high velocities on galactocentric orbits similar to the most ancient stars born in the Galactic bulge and the halo, (2) those that move in the Galactic disk along paths that resemble the circular orbits of massive stars formed in the disk, and (3) high and intermediate mass X-ray binaries running away from their parent regions of star formation. Here we discuss some of the cases studied.
\end{abstract}

\section{Introduction}

The large transverse motions of neutron stars (NS's) in the plane of the sky are believed to result from kicks imparted in natal supernova (SN) explosions. SN explosions are usually invoked in models of the core collapse of massive stars onto black holes (BH's), but until present there have been few observations that can constrain the models of the physical processes by which stellar-mass black holes are formed.

The velocity in three dimensions and the galactocentric orbit can be used to gain insight into this issue, tracking the compact object back to its birth place and constraining the energy of any putative natal kick. Compact microquasar jets are ubiquitous among accreting black holes and neutron stars, and their motion in the plane of the sky can be followed with high precision by astrometry at radio wavelengths with Very Long Baseline Interferometry (VLBI). The proper motion can also be determined by astrometry of the donor star at optical wavelengths. Optical and IR spectroscopy of the companion star provides the line of sight velocity of the system. Knowing the distance either from VLBI or from the properties of the donor star, the Galactic orbit of the X-ray binary can be computed using a Galactic potential model.

Here we summarize the results of a series of papers on individual objects, on which we search for some new constrains to the physical models that describe the formation of stellar-mass black holes and neutron stars.

\section{Results}

The low mass X-ray binaries XTEJ1118+480 (Mirabel et al. 2001) and ScoX-1 (Mirabel \& Rodrigues 2003a) move both in highly eccentric orbits out of the Galactic plane, with space velocities of $150 \mathrm{~km} \mathrm{~s}^{-1}$ and $215 \mathrm{~km} \mathrm{~s}^{-1}$, respectively. Sco X-1 comes from the Galactic bulge in a chaotic orbit; XTE J1118+480 travels in an orbit 
similar to that of the globular clusters. These systems move just like the most ancient objects in the Galactic bulge and halo.

The proper motion of the high mass x-ray binary (HMXB) GRO J1655-40 was measured with HST. It has an intermediate stellar mass black hole of $\mathrm{M}_{B H}=5.4 \mathrm{M}_{\odot}$ and a secondary star of $\mathrm{M}_{2}=1.45 \mathrm{M}_{\odot}$ in an eccentric orbit in the Galactic plane. Presently it has a space velocity of $\mathrm{V}=112 \mathrm{~km} \mathrm{~s}^{-1}$. It was formed out in the disk and probably received a kick from a $\mathrm{SN}$ explosion (the space velocity is $\mathrm{V}=112 \mathrm{~km} \mathrm{~s}^{-1}$ ). It is the first stellar mass black hole candidate with evidence of a kick. It may be the missing link between the formation mechanisms of NS and BH.

Cygnus X-1 (Mirabel \& Rodrigues 2003b) and GRS 1915+105 (Israelian et al. 1999), harbor high mass stellar BH's $\left(10\right.$ and $\left.14 \mathrm{M}_{\odot}\right)$ and move in circular orbits, like massive disk stars. The kinematics of Cygnus X-1 implies that the progenitor of the $\mathrm{BH}$ was a $>25$ $\mathrm{M}_{\odot}$ star, which collapsed via low energy supernova or prompt collapse. LS 5039 (Ribó et al. 2002) is a high mass X-ray binary that move away from its birth place (V=162 $\mathrm{km} \mathrm{s}^{-1}$ ), has an intrinsic binary orbit and a Galactocentric orbit of high eccentricity, suggesting that they received a kick in a supernova explosion. The results on Cygnus X1, GRS 1915+105 and LS 5039 are consistent with the theoretical model in which the energy of the SN explosion falls as a function of the masses of the stellar progenitors (Fryer \& Kalogera 2001).

The high mass X-ray binary LSI $+61^{\circ} 303$ (Mirabel \& Rodrigues 2004) has formed in the open cluster IC1805 1.7 Myr ago. Its space velocity relative to IC1805 (V=27 $\left.\mathrm{km} \mathrm{s}^{-1}\right)$ suggests that a very low amount of mass was ejected in the $\mathrm{SN}$ explosion $>3.5 \mathrm{M}_{\odot}$ ). There are indications that the progenitor of the compact object in LSI $+61^{\circ} 303$ begun its life with $>60 \mathrm{M}_{\odot}$, and should have lost $90 \%$ of its mass before the explosion, also consistent with the Fryer \& Kalogera (2001) model.

\section{References}

Mirabel, I. F., Dhawan, V., Mignani, R., Rodrigues, I., \& Gugielmetti, F. 2001, Nature, 413, 139

Mirabel, I. F., \& Rodrigues, I. 2003a, A\&A, 398, L25

Mirabel., I. F., \& Rodrigues, I. 2003b, Science, 300, 1119

Israelian, G., Rebolo, R., Basri, G., Casares, J., \& Martin, E. L. 1999, Nature, 401, 142

Ribó, M., Paredes, J. M., Romero, G. E., Benaglia, P., Martí, J., Fors, O., \& García-Sánchez, J. 2002, A\&A, 384, 954

Fryer, C. L., \& Kalogera, V. 2001, ApJ, 554, 548

Mirabel, I. F., \& Rodrigues, I. 2004, A\&A, submitted 